\title{
The Microcalorimeter for Industrial Applications
}

Del Redfern and Joe Nicolosi

EDAX Inc.,

91 McKee Dr.,

Mahwah, NJ 07430 USA

\section{Jens Höhne}

CSP Cryogenic Spectrometers

$\mathrm{GmbH}$,

Munich, Germany

\section{Rainer Weiland and \\ Birgit Simmnacher}

Infineon Technologies AG,

Munich, Germany

and

\section{Christian Hollerich}

Technische Universität München, Munich, Germany
To achieve the dramatic increases in $\mathrm{x}$-ray spectral resolution $(<20 \mathrm{eV}$ at $1.5 \mathrm{k} \mathrm{eV})$

desired by market segments such as

the semiconductor industry, NIST

developed a transition-edge sensor (TES)

microcalorimeter. To bring this exciting,

yet demanding, new technology to the industrial users, certain criteria must be

addressed. Aspects of resolution, cooling and hold time, count rates as well as vibrations are considered. Data is presented

to the present efforts to handle these

issues as well as discussing development plans for the future.

Key words: cryogen-free cooling; energy resolution; microcalorimeter energy dispersive; transition-edge sensor; $\mathrm{x}$-ray microanalysis; $\mathrm{x}$-ray spectrometer.
Accepted: August 22, 2002

Available online: http://www.nist.gov/jres

\section{Introduction}

The requirement for improvement in $\mathrm{x}$-ray detector technology has been a major necessity for the semiconductor industry in their long term goal to address the analytical requirements of particles down to $35 \mathrm{~nm}$ as discussed in the 1997 National Technology Roadmap for Semiconductors (NTRS) [1]. To achieve these analytical requirements on the scanning electron microscope, low excitation voltages must be used, and therefore low energy $\mathrm{x}$-ray spectrometry is a necessity. An increase in resolution of the energy dispersive x-ray spectrometer by an order of magnitude is required. To achieve this dramatic increase in resolution $(<20 \mathrm{eV}$ at $1.5 \mathrm{k} \mathrm{eV})$, NIST developed a transitionedge sensor (TES) microcalorimeter [2]. The NIST energy dispersive microcalorimeter spectrometer for x-ray microanalysis used cryogens and an adiabatic demagnetization refrigerator (ADR) to achieve the working temperatures $(<100 \mathrm{mK})$ of the superconducting TES. 
To bring this exciting technology to industrial applications, EDAX INC ${ }^{1}$ and CSP (Cryogenic Spectrometers) $\mathrm{GmbH}$ established a partnership to develop a commercially available microcalorimeter and installed the first beta unit at Infineon Munich.

\section{Requirements for Industrial Applications}

To move from a research tool to a commercially accepted routine analytical tool there are certain criteria that need to be met. These criteria include energy resolution, cooling and available hold time, count rates, and vibration. Development work has been completed (and further developments planned) on each criterion to achieve the requirements for industrial applications.

\subsection{Resolution}

The semiconductor industry has a requirement for small particle $(<100 \mathrm{~nm})$ analysis to determine their composition [1]. Typical applications within the industry [3] are to establish the simultaneous presence of $\mathrm{Ti}$ and $\mathrm{N}, \mathrm{Si}$, and $\mathrm{W}$, or $\mathrm{Si}$ and $\mathrm{Ta}$ in particles with nanoscale dimensions. To achieve these requirements, low excitation voltages have to be applied, thus forcing the analyst to use low energy $\mathrm{x}$-ray spectra to confirm the presence of the elements of interest. Therefore the first criterion an energy dispersive microcalorimeter x-ray spectrometer must be able to achieve is a resolution of $<20 \mathrm{eV}$ at $1.5 \mathrm{k} \mathrm{eV}$.

Figure 1 shows a spectrum of $\mathrm{Al}$ measured on the microcalorimeter with an energy resolution of $13 \mathrm{eV}$. Figures 2, 3, and 4 demonstrate the ability of the microcalorimeter to resolve important peak interference problems: N K and Ti L $\alpha$ (Fig. 2), Si K from W M (Fig. 3) and Si K from Ta M (Fig. 4). The red lines in the spectra are comparisons against a typical $\mathrm{Si}(\mathrm{Li})$ energy dispersive detector.

\subsection{Cooling and Hold Time}

The second criterion is cooling. The NIST microcalorimeter employed liquid nitrogen and liquid helium to achieve a temperature of $4 \mathrm{~K}$. An ADR [4] was then used to take the temperature down to the operating range of the TES of $<100 \mathrm{mK}$. To bring the microcalorimeter into a semiconductor manufacturing environment where the use of liquid cryogens is

\footnotetext{
${ }^{1}$ NIST disclaimer: Certain commercial equipment, instruments, or materials are identified in this paper to foster understanding. Such identification does not imply recommendation or endorsement by the National Institute of Standards and Technology, nor does it imply that the materials or equipment are necessarily the best available for the purpose.
}

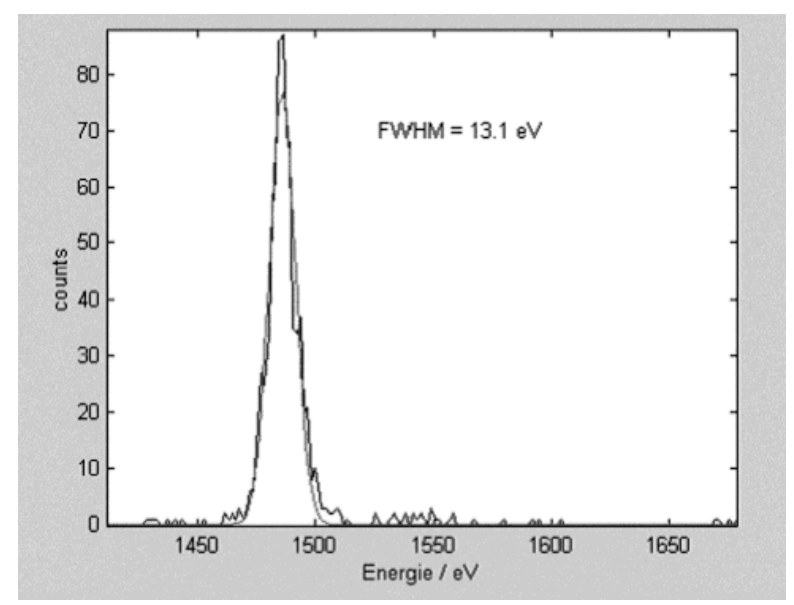

Fig. 1. Aluminum spectrum with $13 \mathrm{eV}$ resolution (smooth trace $=$ fitted peak).

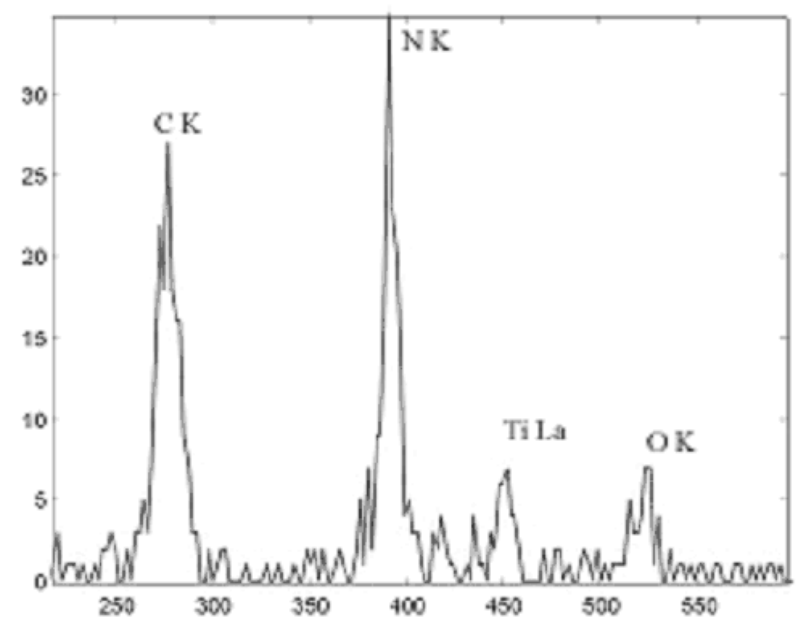

Fig. 2. Ti-N spectrum with $\mathrm{C}$ and $\mathrm{O}$ present.

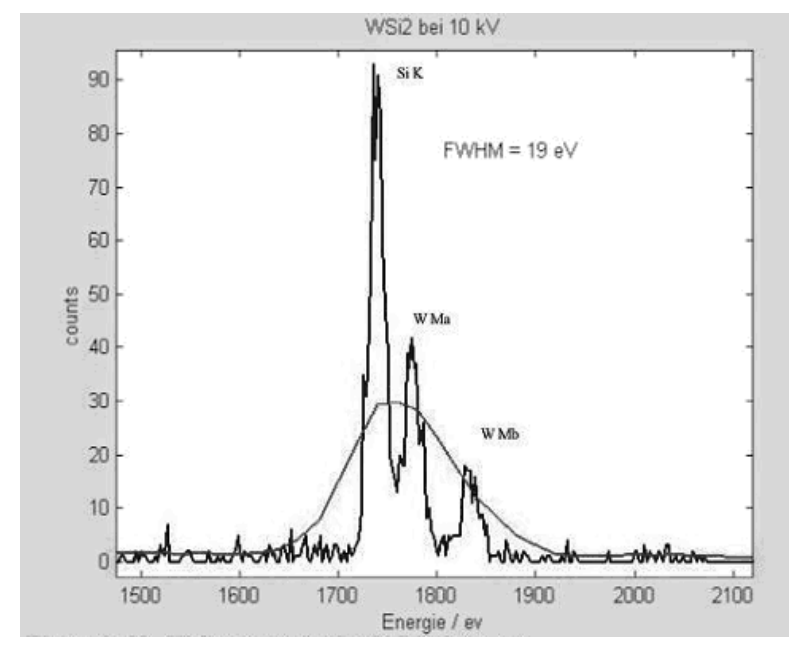

Fig. 3. Si-W spectrum (smooth trace $=$ fitted peak). 


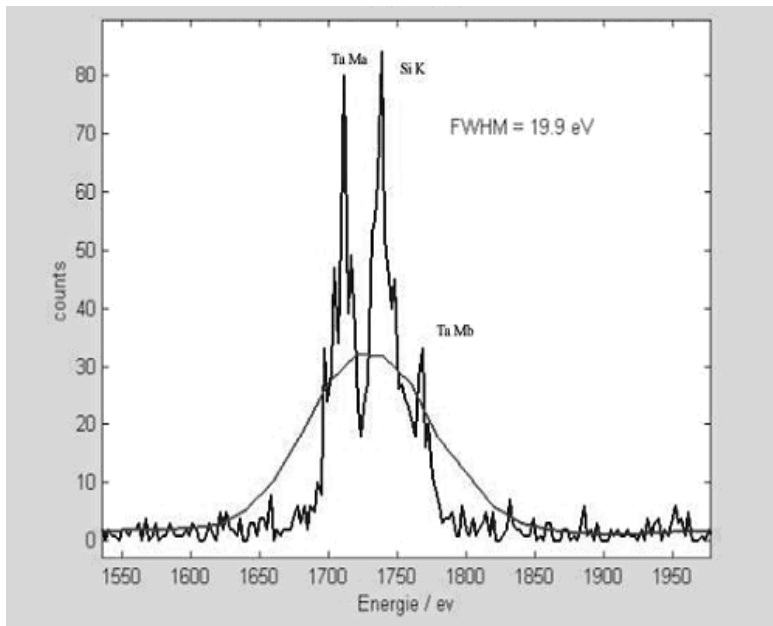

Fig. 4. Si-Ta spectrum (smooth line $=$ conventional $\mathrm{Si}(\mathrm{Li}) \mathrm{EDS})$.

discouraged, CSP developed the pulse tube cooling system, a mechanically cooled device that will generate temperatures to $<4 \mathrm{~K}$ without the use of cryogens. The CSP microcalorimeter employs the ADR in the final cooling stage to reach temperatures of $<100 \mathrm{mK}$. The ADR [4] has a working period at low temperature of approximately $10 \mathrm{~h}$. After this time the ADR has to be recharged (this procedure takes approximately $1 \mathrm{~h}$ ) and then it is available for further use. A major requirement for the industrial tool is automation and longer available working time of the system. The mechanically cooled microcalorimeter can be programmed to begin the recharging cycle of the ADR at a pre-programmed time. The working time of the salt pill can be extended to over $24 \mathrm{~h}$ by employing the "extended hold time" (EHT) unit. The use of the "extended hold time" unit enables the analyst to have "full" availability of the microcalorimeter should they find any one hour free during the day. Figure 5 shows the ADR working temperature with the EHT unit employed with a working temperature of $30 \mathrm{~h}$.

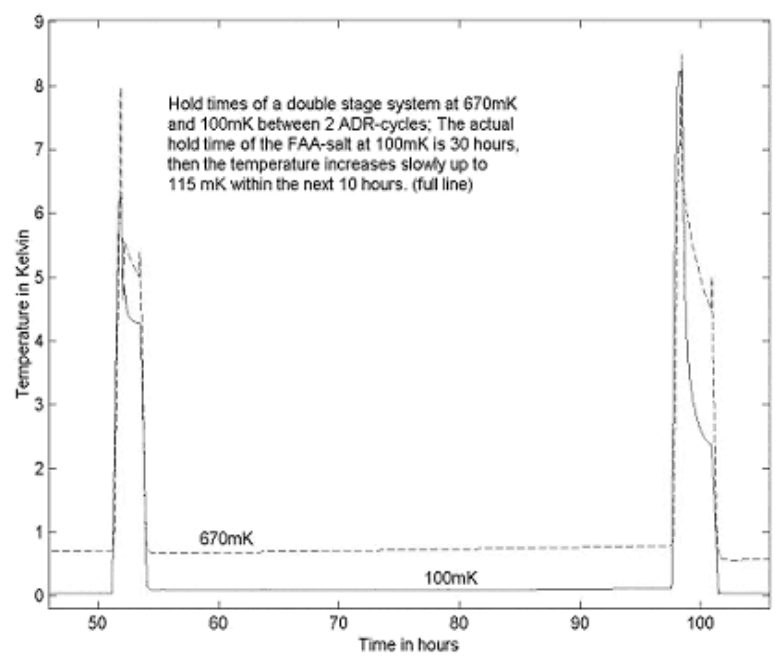

Fig. 5. Cooling curve with extended hold time unit.

\subsection{Count Rates}

The acquisition times required by the semiconductor industry are $<60 \mathrm{~s}$ per particle. The detector (absorber) area is only $400 \mu \mathrm{m}^{2}$ at a working distance of $15 \mathrm{~mm}$. The typical counts per second achievable using the present configuration of the microcalorimeter are around 6 counts per second (using $5 \mathrm{kV}, 700 \mathrm{pA}$ ). This may seem very low but considering the background levels are extremely low and the resolution of the detector very high most of the counts collected are very meaningful. A typical acquisition time is around $3 \mathrm{~min}$. Within $1 \mathrm{~min}$ an experienced operator can determine the presence of the elements of interest, but $3 \mathrm{~min}$ of spectral accumulation is required for $100 \%$ confidence.

To achieve the required count per seconds there are four areas that can be evaluated. The first is the simplest, which is to match the collimator with the detector size. The present system has a circular collimator and a square detector, and these will be matched in the future. The second modification will be to improve the solid angle of the detector, which at present is not optimum. These two minor changes to the configuration are expected to increase the count rate by a factor of 6 . This improvement should bring the acquisition time below $60 \mathrm{~s}$, but two other modifications are possible. The first is the use of polycapillary optics [5]. These optics utilize high-efficiency grazing-angle x-ray reflections to deflect $\mathrm{x}$ rays over a wide angle. Using these optics an increase in counts per second of over 10 fold should be possible. The last option to increase the counts per second is to use a larger-area absorber (this is a long term development plan and will not be considered for this generation of microcalorimeter).

\subsection{Vibration}

A high resolution SEM has strict criteria for vibration requirements. The CSP / EDAX Polaris microcalorimeter energy dispersive $\mathrm{x}$-ray spectrometer weighs approximately $50 \mathrm{~kg}$ and is mounted to the chamber via a port adapter. The only moving part of the microcalorimeter is the rotating valve that stands on top of a pole and is connected to the system via thin tubing. Vibration measurements have been taken, and Figs. 6 and 7 show the effect of the mechanical cooling on the SEM of an image taken at 100000 magnification. The image with the cooler on shows a slight effect of vibration.

The last issue to resolve for the microcalorimeter energy dispersive $\mathrm{x}$-ray spectrometer to be completely acceptable for industrial applications is a noise issue. The rotating valve (located on the pole at the side of the Polaris system shown in Fig. 8) produces a pinging sound at low frequency which will be reduced to background values with appropriate shielding. 


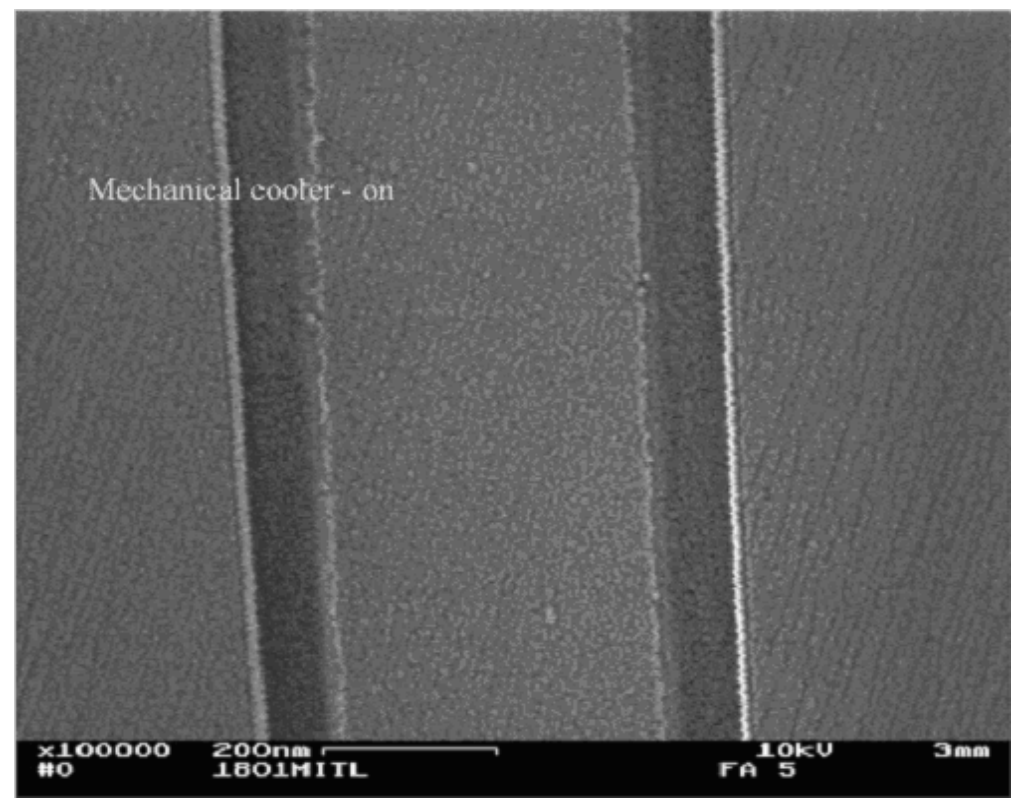

Fig. 6. SEM image at $100000 \times$ magnification with cooler on. Note vibration effects at bright edges and loss of fine-scale details.

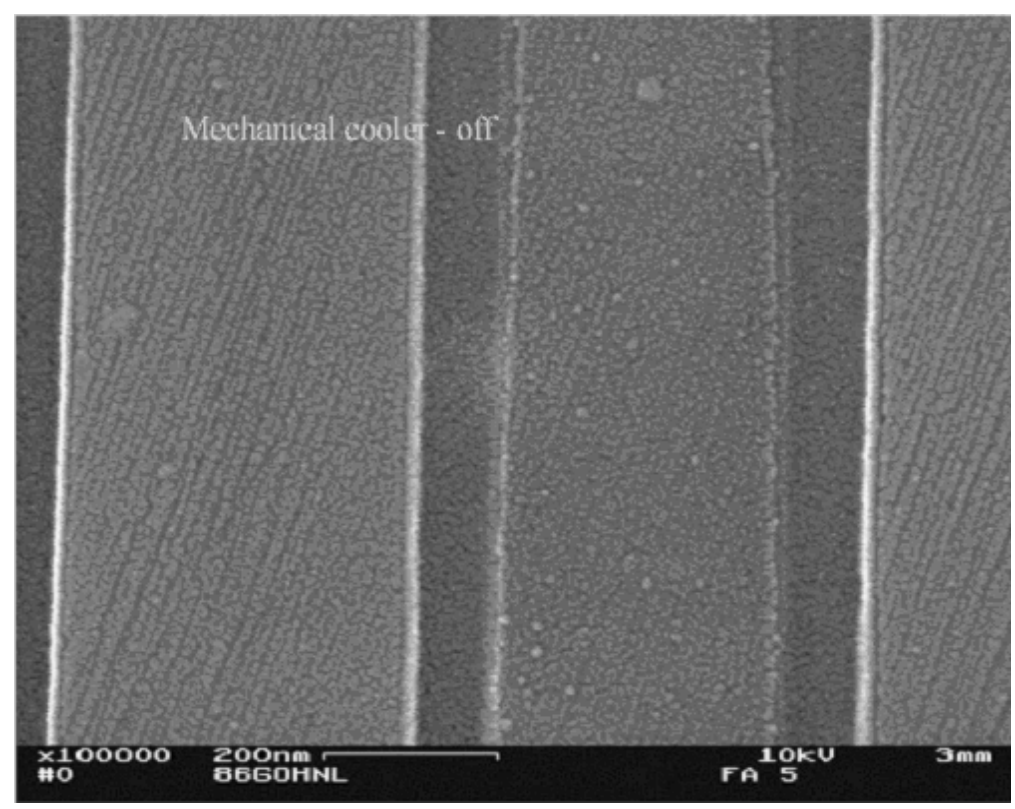

Fig. 7. SEM image at $100000 \times$ magnification with cooler off. Note improved edge image and recovery of fine-scale details. 


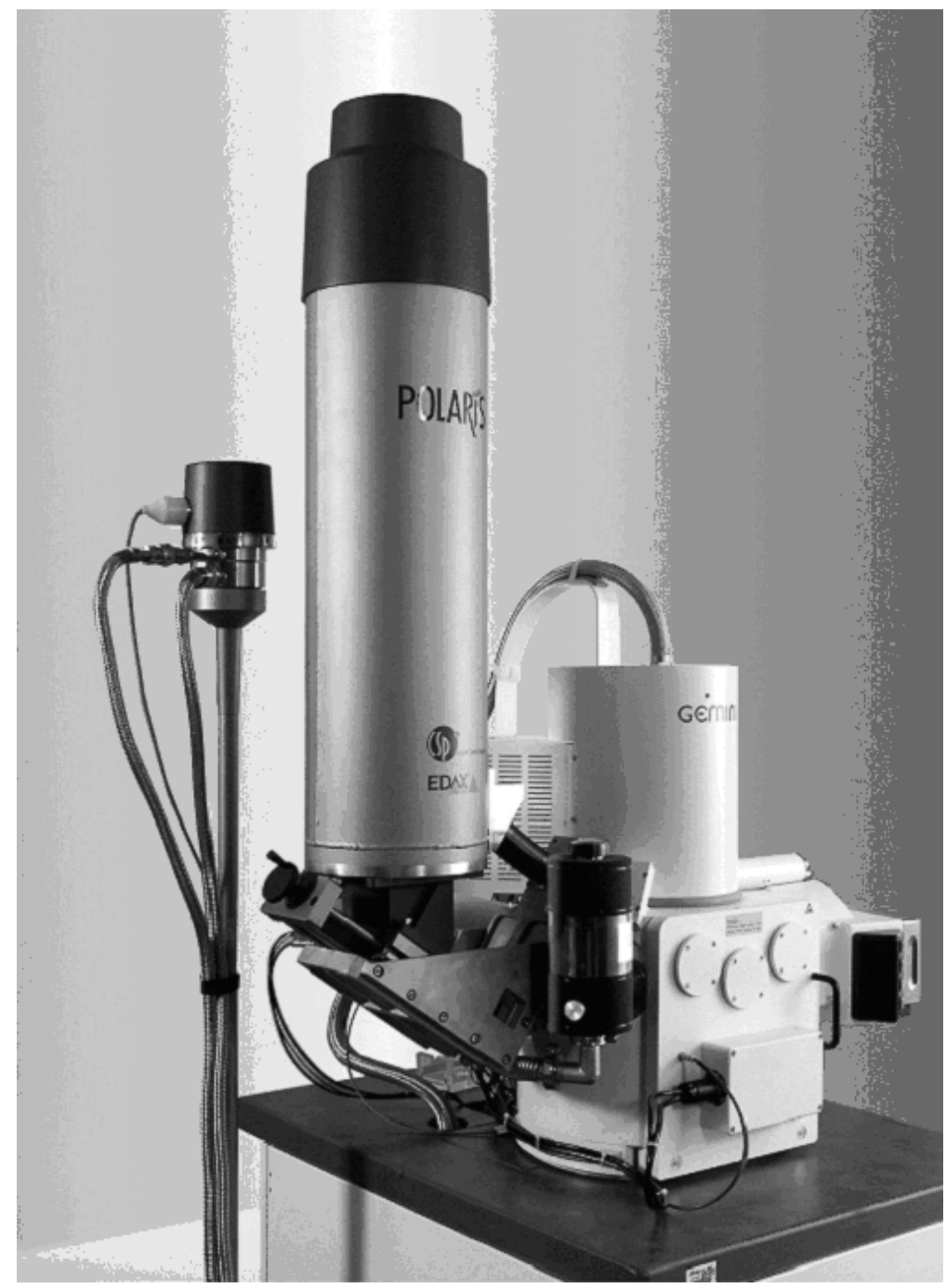

Fig. 8. Polaris microcalorimeter energy dispersive x-ray spectrometer.

\section{Conclusion}

Today the microcalorimeter energy dispersive $\mathrm{x}$-ray spectrometer installed at Infineon, Munich is able to acquire a spectra with a resolution of $15 \mathrm{eV}$ at $1.5 \mathrm{k} \mathrm{eV}$ within $3 \mathrm{~min}$, has a hold time of $8 \mathrm{~h}$, and is mechanically cooled. There are plans to further develop the system to ensure it satisfies all criteria for the industrial applications. These plans are designed to increase the count rate of the beta unit and include modifications to the geometry, i.e., the collimator and solid angle, testing of the polycapillary optics and alig nment issues, and largerarea X-ray absorbers. The EHT unit will enable the microcalorimeter to be available for periods $>24 \mathrm{~h}$.
Vibration issues still have to be resolved to ensure vibration free operation at magnifications of $200000 \times$. For example, the noise of the rotating valve has to be reduced to background levels. Once all these modifications and improvements have been completed, we will see the microcalorimeter energy dispersive x-ray spectrometer commercially available for industrial applications.

\section{References}

[1] The National Technology Roadmap for Semiconductors, The Semiconductor Industry Association, 4300 Stevens Creek Boulevard, Suite 271, San Jose, CA 95129 (1997). 
[2] D. A. Wollman, G. C. Hilton, K. D. Irwin, and J. M. Martinis, Proceedings of Microscopy and Microanalysis 1996, pp. 488-489

[3] C. Boit, R. Weiland, A. Olbrich, U. Mühle, and B. Simmnacher, Failure Analysis Concepts for Microelectronics Technologies and Manufacturing of the Future, Edinburgh EMM-Conference Proceedings (2001).

[4] J. Höhne, M Altman, G. Angloher, P. Hettl, J. Jochum, T. Nüßle, S. Pfnür, J. Schnagl, M. L. Sarsa, S. Wänninger, F. v. Feilitzsch, High Resolution X-Ray Spectroscopy Using Iridium/Gold Phase Transition Thermometers, EDXRF Bologna (1998).

[5] D. A. Wollman, S. W. Nam, D. E. Newbury, G. C. Hilton, K. D. Irwin, N. F. Bergen, S. Deiker, D. A. Rudman, J. M. Martinis, Superconducting transition-edge-microcalorimeter $\mathrm{X}$-ray spectrometer with $2 \mathrm{eV}$ energy resolution at $1.5 \mathrm{keV}$, Nucl. Instr. Meth. A 444, 145-150 (2000).

About the authors: Del Redfern is Micro Characterization Tools Product Manager for EDAX Inc., a unit of Ametek Process and Analytical Instruments responsible for handling all matters pertaining to Microanalysis; including strategic product planning, product specification, budget planning, marketing programs, sales support and financial planning. Before joining EDAX, Del worked for Philips Analytical, his last position there was XRF RegionalMarketing Manager.

Christian Hollerith studied physics at the Technical University of Munich. He received his diploma in 2001 with a thesis on microcalorimeter detectors. Since then he has been working as a doctoral student with the Technical University of Munich on the POLARIS microcalorimeter detector, in collaboration with Infineon Technologies AG and VeriCold Technologies GmbH.

Jens Höne studied physics in Munich. He received his PhD with a thesis on superconducting detectors for astro particle physics applications in 1998. He started his own business to develop the POLARIS microcalorimeter detector. Currently he is CEO of the newly founded VeriCold Technologies GmbH which has taken over the development and production of the POLARIS system, as well as various cooling technologies in March 2002.

Joseph A. Nicolosi, PhD, is director of Technology for EDAX Inc., a unit of Ametek Process and Analytical Instruments responsible for $R \& D$ of solid state $x$-ray detectors and related detecting unit technologies including: high vacuum cryogenics, pulse processing electronics, application software and technical support. Related research activities have included: $x$-ray diffraction physics and development of $x$-ray methods and instrumentation for materials analysis. During 30 years in these product areas Joe has co-authored 7 patents, 22 journal publications and 18 conference presentations. 